\title{
Fimbria/fornix lesions facilitate the learning of a nonspatial response task
}

\author{
DOUGLAS B. MATTHEWS and PHILLIP J. BEST \\ Miami University, Oxford, Ohio
}

\begin{abstract}
The spatial cognitive map theory of O'Keefe and Nadel (1978) predicts that lesions of the hippocampal system should impair learning on spatial tasks but not learning on nonspatial tasks. However, there is evidence that such lesions can facilitate learning on certain nonspatial tasks. Their theory does not predict such facilitation. Nevertheless, it is reasonable to expect that animals possessing a spatial cognitive map would have an inherent bias to engage a mapping strategy and thus be at a disadvantage on certain nonspatial tasks in comparison with animals without the mapping capacity and bias. In the present study, fimbria/fornix lesions impaired learning on a spatial task, but actually facilitated learning on a nonspatial task of equal difficulty. Thus, brain lesions that interfere with map functioning can facilitate learning on tasks for which a mapping strategy interferes with task solution. The results require a modification of the spatial cognitive map theory.
\end{abstract}

According to O'Keefe and Nadel (1978), the primary function of the hippocampus is to serve as a spatial cognitive map. Nadel (1991) further emphasized that one of the fundamental purposes of the spatial cognitive map is to support spatial memory, regardless of task demands. The theory predicts that lesions to the hippocampal system will impair the learning of spatial tasks, presumably because these tasks require a spatial cognitive map. Furthermore, the theory predicts that lesions to the hippocampal system will not impair the learning of nonspatial tasks, presumably because these tasks do not require a spatial cognitive map.

Several studies have reported findings consistent with these predictions. Morris, Garrud, Rawlins, and O'Keefe (1982) trained rats in the water maze to swim to a submerged platform from several start locations while other rats were trained in the same maze to swim to a visible platform. They found that rats with bilateral hippocampal lesions were impaired in the spatial task in comparison with controls, but that both lesioned and control rats learned the nonspatial task at the same rate. Using a more difficult task, O'Keefe, Nadel, Keightley, \& Kill (1975) trained water-deprived rats to find a water well on a circular runway by using either spatial cues or a single nonspatial cue (a light). They found that fornix lesions

We wish to thank Lynn Nadel, Jerry Rudy, and an anonymous reviewer for insightful comments on an earlier draft of the paper. This work was partially supported by a challenge grant to P.J.B. from the Ohio Board of Regents. Correspondence should be addressed to P. J. Best, 136 Benton Hall, Psychology Department, Miami University, Oxford, OH 45056 (e-mail: pjest@miamiu.acs.muohio.edu). impaired the learning of the spatial task in comparison with the performance of controls but that the two groups did not differ significantly on the nonspatial task.

Surprisingly, some studies have reported that lesions to the hippocampal system may actually facilitate learning on certain nonspatial tasks. Conditioned place learning is facilitated by fornix lesions (White \& McDonald, 1993), and selective fimbria lesions can facilitate object recognition (M'Harzi, Jarrard, Willig, Palacios, \& Delacour, 1991). Thus, lesioning the hippocampal system and destroying the spatial cognitive map may actually facilitate learning on tasks that require the animal to ignore spatial information. Facilitated learning on nonspatial tasks is not predicted by the spatial cognitive map theory. The theory simply does not predict that animals with an intact hippocampus are predisposed to use their spatial cognitive map even when the task requires nonspatial processing.

In light of studies showing enhanced learning following lesions, it appears that Morris et al. (1982) and O'Keefe et al. (1975) failed to demonstrate facilitation because of a floor effect. The nonspatial tasks that they used were too easy. O'Keefe et al. required subjects to learn to navigate to a single light, and Morris et al. required subjects to swim to a visible platform. Thus, it may have been the ease of the task and not the nonspatial aspects that led to the nonsignificant difference in learning rates between lesion and control animals.

We propose a corollary to the spatial cognitive map theory. First, animals with an intact hippocampus, and therefore a spatial cognitive map, will be predisposed to process spatial information even when such processing is incompatible with task demands. Second, animals 
with a lesioned hippocampal system, and therefore no spatial cognitive map, will show facilitated learning in difficult tasks in which the animal must navigate through space but ignore the corresponding spatial information.

In the present study, we attempted to provide support for such a corollary by investigating the effects of fimbria/ fornix lesions on performance of a spatial and a nonspatial task of sufficient difficulty to protect against a possible floor effect. In addition, the tasks differed only in their reliance on spatial processing. The place (spatial) task requires the subject to navigate to a constant location in the radial arm maze regardless of the starting location, thereby requiring the subject to make a different body response on every trial. The response (nonspatial) task requires the subject to make a constant body response, regardless of the start location. Thus, the subject navigates to a different location on every trial. Note that the response task requires the subject to go to a different place on each trial. Consequently, the animal with a functioning cognitive map (the control animal) should be disadvantaged in the response task.

Both tasks were conducted on an eight-arm radial arm maze. Subjects were trained for seven trials per day, with each trial starting from a different arm, to navigate to either the same arm as determined by distal cues for food reward (place task) or to navigate to the arm $90^{\circ}$ to the left of the start arm, regardless of the distal cues, for food reward (response task). The place task requires the animal to monitor spatial cues and perform a different response on each trial, whereas the response task requires the animal to not use spatial cues and instead learn a constant body response.

If O'Keefe and Nadel (1978) are correct in assuming that animals with a hippocampus can form a spatial cognitive map, and if the corollary that they are predisposed to process space is correct, then lesions of the fimbria/ fornix will impair the learning of the place task but facilitate the learning of the response task. Presumably, the spatial cognitive map formed by the control rats will interfere with learning the response task. Lesioned animals cannot form a spatial cognitive map, and therefore their learning of the response task will not be interfered with. In fact, the fimbria/fornix lesions should facilitate learning of the response task.

\section{METHOD}

\section{Subjects \\ Forty-one male rats approximately 45 days of age from Charles- River were housed in hanging steel cages, on a 12:12-h light:dark cycle (lights on at 7:00 a.m., handling and training between 9:00 a.m. and noon) in an approved animal colony. After 2 weeks of ad-lib food and water, the availability of food was restricted to $1 \mathrm{~h}$ per day. The an- imals were weighed daily to ensure that they maintained at least $80 \%$ of their free-feeding weights; the animals were never water deprived. \\ Surgical Procedure \\ The animals received either bilateral radio-frequency fimbria/fornix lesions at four sites or sham lesions. Surgery occurred 1 week follow- ing the beginning of the restricted feeding schedule. \\ Prior to surgery, the subjects were injected i.p. with $.05 \mathrm{cc}$ atropine and $.45 \mathrm{mg} / \mathrm{kg} \mathrm{Nembutal}$; booster doses were given as needed. The}

subjects were placed, flat head, in a sterile stereotaxic instrument; the skull was drilled, and the dura was torn and removed. The lesions were made with a Radionics Research RF Lesion Generator System, Model RFG-4A. The electrode tip was maintained at $70^{\circ} \mathrm{C}$ for $1 \mathrm{~min}$ at $.3 \mathrm{~mm}$ posterior to bregma, $.7 \mathrm{~mm}$ lateral to midline, and $4.0 \mathrm{~mm}$ and $4.2 \mathrm{~mm}$ ventral of dura; $.8 \mathrm{~mm}$ posterior to bregma, $1.7 \mathrm{~mm}$ lateral to midline, and $4.0 \mathrm{~mm}$ and $4.4 \mathrm{~mm}$ ventral of dura (Eichenbaum, Stewart, \& Morris, 1990). The incision was then closed, and the animal was returned to its home cage. The subjects in the sham condition underwent an identical procedure, except that the dura remained intact. The subjects were weighed daily, and the state of postsurgical healing was monitored for a period of 7 days.

\section{Behavioral Procedures: Adaptation}

Before pretraining, the subjects were exposed to an elevated fourarm radial maze. The maze was $91 \mathrm{~cm}$ above the floor, with four 51$\mathrm{cm}$-long, $11.5-\mathrm{cm}$-wide arms arrayed at right angles. The maze was located at the center of a testing room that contained several extramaze cues (a door, several bookcases, hanging black curtains to one side, and a VCR and monitor).

Subjects were allowed to explore the maze for $4 \mathrm{~min}$, twice per day for 3 days. Pieces of Froot Loops cereal were scattered throughout the maze and in metal feeding bowls located at the end of each arm. The number of arms entered and the number of pieces of cereal eaten were recorded.

\section{Pretraining}

Subjects were pretrained in a different testing room on an elevated eight-arm radial arm maze. The maze was $91 \mathrm{~cm}$ above the floor and had 51-cm-long and 11.5 -cm-wide arms that radiated from a central platform at intervals of $45^{\circ}$. The central platform was an octagonal chamber $25 \mathrm{~cm}$ in diameter and $32 \mathrm{~cm}$ high. The chamber contained eight guillotine doors. The doors were aligned in front of each radial arm. The arrangement allowed the experimenter to lower a door to block access to any arm. A metal feeding bowl at the end of the goal arm contained one-quarter piece of Froot Loops cereal. The testing room contained several extramaze cues (a table, a window, a door, and an overhead bar).

During this phase, the animals were given seven forced trials per day, with the start and goal arms different on every trial so that no place-learning strategy or response-learning strategy was reinforced. To accomplish this, the doors on each trial were aligned so that only the ones corresponding to either the start arm or the goal arm were up while all other doors were in the down position. Thus the animal was not allowed to choose a goal arm but instead was forced to select the correct arm on the basis of the juxtaposition of the guillotine doors. The order of the start/goal arms was counterbalanced, so that each arm would be used as a start arm only once per day and the serial order of the arms differed over days. The pairing of the start/goal arms caused the angular relationship between the two arms to vary between $45^{\circ}$ and $180^{\circ}$ (straight alley). Also, the direction of the angular relationship varied with reference to the start arm: the goal arm was not consistently to the right or the left of the start arm. Thus, the subject was trained to run for food reward and not to learn a spatial configuration of distal cues or a particular response for successful navigation of the maze.

During pretraining, the experimenter arranged the doors in the proper configuration and then placed the animal on the start arm facing away from the central chamber. When the subject obtained the reward, the experimenter recorded the running latency. Between trials, the animal was placed in a stainless steel bolding chamber. The experimenter then rearranged the guillotine doors and began the next trial.

Subjects were pretrained for 14 days.

\section{Training}

Following pretraining, animals in each surgical condition were randomly assigned to the place or the response task. Thus there were four groups: hippocampal lesion/place, 10 animals; hippocampal lesion/ response, 12 animals; control/place, 9 animals; and control/response, 10 animals.

Training occurred in a different room, with seven trials per day. A novel eight-arm radial arm maze was used for the training procedure. It had the same dimensions as did the maze used in pretraining. In ad- 
dition, a 5-cm ledge of clear Plexiglas was attached to the sides of each $\mathrm{arm}$. The testing room provided several distinct distal cues (a blackened window, door, book shelf, VCR, camera, table, two posters, and the experimenter).

In the place condition, the location of the goal arm was constant in relation to both the particular arm used and the extramaze cues. The position of the start arm was counterbalanced so that each arm (except the goal arm) was used once as a start arm per day, with a different serial order on each day.

In the response condition, the position of both the start arm and the goal arm varied over trials, but the angular relation between start and goal arms remained constant. The goal arm was always $90^{\circ}$ to the left of the start arm. The possible start arms were always counterbalanced for both conditions.

On each trial, the subject was placed at the end of the start arm, facing away from the center of the maze. Running latency and arm selection were recorded. If the animal selected the correct arm, the experimenter removed the animal from the maze, placing it in a stainless steel holding cage. The experimenter then baited the appropriate goal arm and proceeded with the next trial.

If the animal selected an incorrect goal arm, the experimenter removed the animal from the maze and then lowered each guillotine door (between the central chamber and the radial arms) except for the doors facing the start and goal arms. The animal was then placed on the start arm and the running latency was recorded. The animal was then returned to the holding cage, all guillotine doors were raised, and the next trial was begun.

At the conclusion of a session, the subject was returned to its home cage and given free access to food for $1 \mathrm{~h}$.

The animals were trained for 11 days.

\section{Histology}

When behavioral training was completed, the subjects were deeply anesthetized with Nembutal $(1.0 \mathrm{cc}$, i.p.) and perfused with a $10 \%$ solution of formalin. Each brain was removed and placed in a $10 \%$ formalin/sucrose solution until the brain sank. Each brain was then frozen and sliced at $36 \mu \mathrm{m}$. The slices were observed under a light microscope, and a record was made of the size and location of each lesion, which was then compared with the appropriate brain section in Paxinos and Watson's Rat Brain in Stereotaxic Coordinates.

Reconstructed lesions indicate that the fibers of the fornix were damaged in all animals, with the maximum lesion damaging roughly $85 \%$ of the fornix while the smallest lesion damaged approximately $50 \%$ of the fornix. Small additional damage included the stria terminals, lateral dorsal thalamus, triangular septal nuclei, and the lateral septal nuclei.

\section{RESULTS}

During adaptation, lesioned animals entered more total arms than sham animals when Days 1 and 2 were combined ( $t=2.48, p<.02$ ), but no difference between conditions was seen on Day $3(t=.06, p>.1)$. Comparisons of Pretraining Day 1 and Day 14 showed that latency decreased significantly for both groups (lesioned, $t=9.83, p<.001$; sham, $t=6.29, p<.001$ ). Lesioned rats had shorter latencies on Pretraining Day 1 than did sham rats $(t=2.62, p<.02)$, but the groups' latencies were almost identical on Pretraining Day $14(t=.22, p>.1)$.

A $2 \times 2$ analysis of variance (ANOVA) examining day to criterion of at least six of seven correct revealed no significant main effects, but there was a significant interaction between lesion and task condition $(F=$ $10.31, p<.003$ ) (see Figure 1 ).

Simple $t$ tests confirm the significant interaction in the ANOVA. Namely, in the place task, lesioned animals reached criterion significantly slower than did the sham

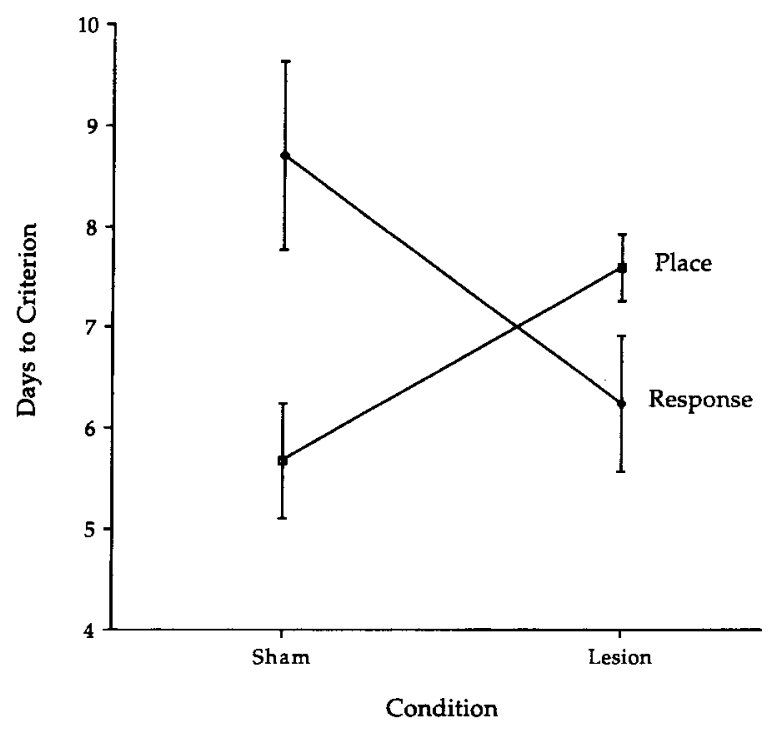

Figure 1. Mean number of days to criterion (six of seven correct choices) on the two tasks by surgical condition (sham and lesion).

animals $(t=2.96, p<.01)$, but in the response task, lesioned animals reached criterion significantly faster than the sham animals $(t=2.16, p<.05)$. Sham animals reached criterion significantly faster in the place task than they did in the response task $(t=2.69, p<.02)$. Lesioned animals reached criterion slightly, but not significantly, faster in the response task than in the place task $(t=1.65, .15>p>.10)$

\section{DISCUSSION}

Fimbria/fornix lesioned animals performed significantly better than sham control animals in the response task and performed significantly worse than sham animals in the place task. The results support O'Keefe and Nadel's (1978) claim that one of the primary functions of the hippocampus is to construct a spatial cognitive map. Furthermore, the results suggest that having an intact hippocampus and therefore a spatial cognitive map produces an inherent bias in the animal to use "space" even if processing space puts the animal at a disadvantage.

The finding that in the response condition, lesioned animals performed significantly better than sham animals is especially significant. O'Keefe et al. (1975) and Morris et al. (1982) reported that lesioned animals did not learn a nonspatial task faster than intact animals. We believe that the nonspatial tasks in their studies were simply too easy to permit a test of the hypothesis that a lesion to the hippocampal system could facilitate nonspatial learning. In the present study, the response task was sufficiently difficult to demonstrate the effect. The results also support our corollary to O'Keefe and Nadel's theory-namely, that intact animals required to ignore the spatial information while moving through space learn slower than lesioned animals. This occurs because the intact animal is biased to process space because of a functioning spatial cognitive map, whereas the lesioned animal suffers from no such bias.

Several other theories attempt to explain hippocampal function. One theory holds that the hippocampus is involved in working memory but not reference memory (Olton \& Papas, 1979). Several studies support the role of the hippocampus in spatial working memory (Jarrard, Okaichi, Steward, \& Goldschmidt, 1984; Olton \& Papas, 1979). However, the tasks used in the present study were both reference memory tasks; subjects always went to the same place for food reward (place task) or always went $90^{\circ}$ to the left for food reward (response 
task). If Olton's theory is correct, lesions should have no effect on either task because both have no working memory component. The results do not support this theory.

A second theory holds that the hippocampus is necessary for combining cues into unique configurations (Sutherland \& Rudy, 1989) Both animals with lesions to the hippocampal system and intact animals can use single cues to form simple associations (e.g., simple classical conditioning), but only intact animals can form configurations (e.g., negative patterning). Thus, if a task requires simple associations, no difference should exist between lesioned and intact animals, but if a task requires configural associations, intact animals should perform better than lesioned animals.

According to Sutherland and Rudy (1989), navigating through space requires configural processing; their theory therefore predicts the results obtained in the place task. However, in its existing form, Sutherland and Rudy's theory does not predict the superior learning of the lesioned animals in the response task. Since the response task can be learned by simply using a simple association (always go $90^{\circ}$ to the left), their theory would predict that lesioned animals should perform as wel as sham animals, but it would not predict that they should be superior.

Sutherland and Rudy (1989) state that "the SAS (simple association system) and CAS (configural association system) interact to determine performance in ways that are presently not well understood" (p. 131). Thus the present results force a modification of the configurationa theory. If the theory is correct, intact animals are biased toward processing configural associations at the expense of simple associations. Further work must be done to determine whether types of configurations (e.g., spatial configurations) are easier for intact animals to learn.

Although the lesioned animals learned the place task slower than intact animals, what mechanism allowed for the lesioned animals to learn the task at all? Allocentric space is determined by the juxtaposition of distal cues, and only intact animals should be able to process this information. However, the cognitive mapping theory predicts that lesioned animals could use a single distal cue or a cluster of distal cues to guide their navigation, a type of taxon navigation. This type of behavior has also been reported by O'Keefe and Conway (1980). It is possible that this type of taxon navigation allowed the lesioned animals to learn in the place task, but at a slower rate than if they had been able to use a cognitive map.

The layout of the distal cues in the experimental testing room was such that a large, black-shaded window was approximately $15^{\circ}$ to the right of the goal arm. Furthermore, the experimenters, who were blind to the lesion condition, reported that animals "seemed to be looking for a cue when in the center of the maze." Post hoc comparisons of research notes revealed that this was the case for the majority of the lesioned place animals and was reported for only 1 sham place animal.

We believe that this result is intuitive. For example, imagine that your new laboratory is located across the hall from your new office. To the east of your office is the coffee room. In the morning you leave your office for a cup of coffee; in doing so, you could learn to either go east or turn left for coffee. Later in the day, you decide to leave your laboratory for coffee. What rule would you use? We predict you would go east (turning right) instead of turning left. But, more importantly, if we could rig the situation so that the coffee room was always to the left of the room you were exiting, you would be very confused.

\section{REFERENCES}

Eichenbaum, H., Stewart, C. \& Morris, R. G. M. (1990). Hippocampal representation in place learning. Journal of Neuroscience, 10, 3531-3542.

JarRaRd, L. E., OKaichi, H., Steward, O., \& Goldschmidt, R. B. (1984). On the role of hippocampal connections in the performance of place and cue tasks: Comparisons with damage to hippocampus. Behavioral Neuroscience, 98, 946-954.

M'Harzi, M., Jarrard, L. E., Willig, F., Palacios, A., \& DelaCoUR, J. (1991). Selective fimbria and thalamic lesions differentially impair forms of working memory in rats. Behavioral \& Neural Biology, 56, 221-239.

Morris, R. G. M., Garrud, P., Rawlins, J. N. P., \& O'KeEFe, J. (1982). Place navigation impaired in rats with hippocampal lesions. Nature, 297, 681-683.

NADEL, L. (1991). The hippocampus and space revisited. Hippocampus, 1, 221-229.

O'KeEFE, J., \& CONWAY, D. H. (1980). On the trail of the hippocampal engram. Physiological Psychology, 8, 229-238.

O'KeEFE, J., \& NADEL, L. (1978). The hippocampus as a cognitive map. Oxford: Oxford University Press, Clarendon Press.

O'Keefe, J., NAdEl, L., Keightley, S., \& Kill, D. (1975). Fornix lesions selectively abolish place learning in the rat. Experimental Neurology, 48, 152-166.

Olton, D. S., \& PAPAS, B. C. (1979). Spatial memory and hippocampal function. Neuropsychologia, 17, 669-682.

Paxinos, G., \& Watson, C. (1986). The rat brain in stereotaxic coordinates (2nd ed.). San Diego: Academic Press.

Sutherland, R. J., \& Rudy, J. W. (1989). Configural association theory: The role of the hippocampal formation in learning, memory, and amnesia. Psychobiology, 17, 129-144.

White, N. M., \& McDonald, R. J. (1993). Acquisition of a spatial place preference is impaired by amygdala lesions and improved by fornix lesions. Behavioural Brain Research, 55, 269-281.

(Manuscript received June 6, 1994; revision accepted for publication September 3, 1994.) 